\title{
GLIOBLASTOMA MULTIFORME METABOLISM: FUEL TO THE FLAME
}

Preethi Sridharan, PhD Scholar

\author{
Central Inter-Disciplinary Research Facility,
}

Sri Balaji Vidyapeeth, Mahatma Gandhi Medical College and Research Institute Campus

Pillaiyarkuppam, Puducherry - 607403, India

E-mail: preethiresearcher@gmail.com

i

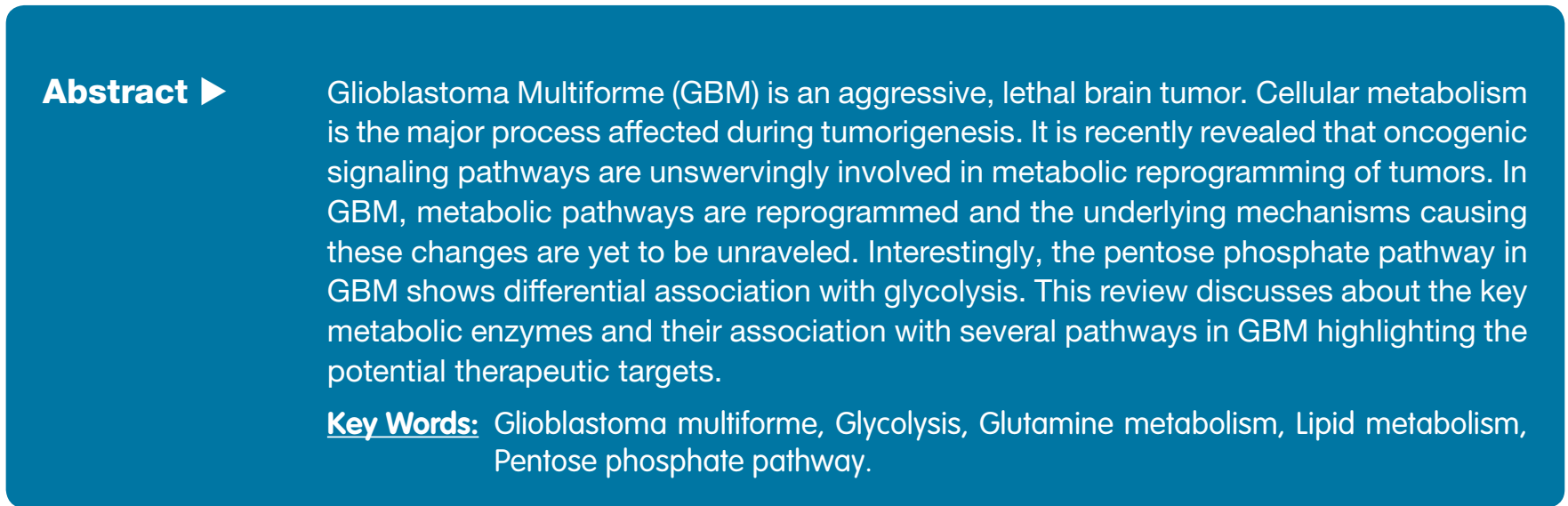

\section{Introduction}

GBM, the most aggressive of the gliomas, is a collection of tumors arising from glial cells or their precursors within the brain. The most common grading system uses the scale of I to IV, of which grade, grade $\mathrm{I}$ is benign, grade II is low grade glioma whereas, grade III and IV are considered to be high grade and malignant. GBMs draw significance because of their poor prognosis (less than a year). The histological features that distinguish GBM from other grades are the presence of necrosis and increased vasculature around the tumor. GBM is characterized by a heterogeneous cell population which makes it genetically unstable, highly infiltrative, angiogenic and resistant to chemotherapy 1. Moreover, GBMs show altered cell metabolism. In recent years, the understanding of the regulation of tumor metabolism has much improved. Evidence show that tumor cells reprogram their metabolism to meet high energy demands which markedly elevate biosynthetic processed and energy production, which in turn promote rapid tumor growth and division (Figure 1). Thus, targeting metabolism has become a promising strategy for cancer treatment ${ }^{2}$.

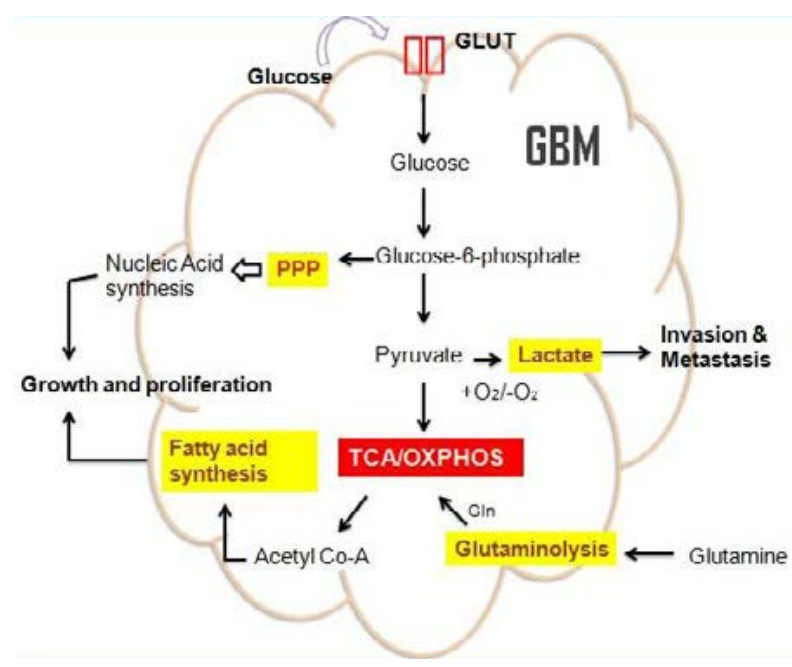

Figure 1: GBM metabolism in a nutshell 


\section{Glycolysis}

The most popular metabolic remodeling described in tumor cells is an increase in glucose uptake, the enhancement of glycolytic capacity and the high lactate production, along with the almost inefficient oxidative phosphorylation even in the presence of high oxygen tension (Warburg effect) ${ }^{3}$. GBMs upregulate glycolysis more than three times that of normal brain tissue as measured by increased lactate: pyruvate ratio. Several in vitro studies have demonstrated large variability in mitochondrial respiration and glucose dependency in cell lines derived from GBM tissues and xenografts. The ability to modulate mitochondrial respiration is an important component to tumor cell survival under hypoxic conditions ${ }^{4-6}$. In addition to enhanced glucose uptake and aerobic glycolysis, GBM cells also exhibit altered glutamine catabolism, particularly within myc expressing cells ${ }^{7,8}$.

\section{Hexokinase}

Hexokinase (HK) catalyzes the first rate limiting step in glycolysis. HK has four isoforms I-IV showing differential tissue expression. In normal human brain and in low-grade gliomas HK-I is predominantly expressed, whereas in high grade gliomas, HK-II is highly expressed $^{9,10}$. Regional variation in HK-II expression is observed in GBMs, showing higher expression in the highly proliferating and apoptosis-resistant perinecrotic central regions, suggesting HK-II may provide a survival and proliferative advantage in vivo, especially within tumor microenvironments. The depletion of HK-II in vitro showed potential inhibition of aerobic glycolysis and promoted normal oxidative glucose metabolism followed by decreased lactate production and increased expression of Oxidative Phosphorylation (OXPHOS) proteins with enhanced $\mathrm{O}_{2}$ consumption. The high affinity kinases $\mathrm{HK} 1$ and $\mathrm{HK}-\mathrm{II}$ are inhibited by excess Glyceraldehyde-6- Phosphate (G6P). They are associated with the mitochondria, are partly responsible for the increased glycolytic tumor activity and are implicated in cell survival. Many inhibitors of HK affecting the glycolytic flux like 2-deoxy-D-glucose (2DG), 3-bromopyruvate and Ionidamine have shown promising effects in preclinical studies.

\section{Pyruvate Kinase}

Pyruvate Kinase (PK) catalyzes the final step of glycolysis converting the phosphoenolpyruvate (PEP) to pyruvate and generating ATP. Four PK forms are identified PKL
\& PKR, PKM1 \& PKM2. M1 and M2 isoforms are produced by alternative splicing containing exon 9 and exon 10 and exhibit different functional activities. PKM1 is constitutively expressed whereas, PKM2 can be regulated by fructose-1,6-bisphosphate. PKM1 expression is down-regulated by the splicing repressors while PKM2 is mainly expressed in cancer cells ${ }^{11}$, and is involved in EGFR signaling pathway in $\mathrm{GBM}^{12}$. Upon its activation by EGFR via phosphorylation at $\operatorname{Ser} 37$ by ERK1/2, PKM2 was shown to translocate from the cytoplasm to the nucleus where it binds to importin ? 5. After entering into the nucleus, PKM2 promotes the transcription of C-MYC which further upregulates glucose transporters (GLUT) and HK-II expression to promote glycolysis ${ }^{13}$. In GBM cells, activation of EGFR by EGF induces PKC? monoubiquitylation that recruits and phosphorylates IKK? ? to promote the interaction of activated Rel A with HIF-1? This complex binds to the PKM2 promoter and transcribes $i^{14}$. It was observed that the PKM1 expressed in normal brain samples exhibited high levels of PK activity whereas a significantly lower activity level was observed in all gliomas including $\mathrm{GBMs}^{15}$. Recently; it was shown that in adult glioma spheroids, PKM2-Oct4 interaction inhibited the stemness property, promoted differentiation and enhanced the sensitivity to cell death ${ }^{16}$.

\section{Isocitrate Debydrogenase}

Isocitrate dehydrogenase (IDH) catalyzes the oxidative decarboxylation of isocitrate to produce ?-Keto Glutarate, generating $\mathrm{NADH}$ in mitochondria or $\mathrm{NADPH}$ in cytoplasm. Five IDH genes have been identified. IDH1 and IDH 2 mutations were first reported in low grade gliomas and secondary GBMs. IDH1 mutation occur $80 \%$ and $85 \%$ of diffuse astrocytomas and secondary GBMs respectively. IDH $1 / 2$ mutants gain new enzyme activity, which catalyzes ?-KG to 2-hydroxyglutarate (2-HG), a metabolite mostly produced by error during normal metabolism and leads to its accumulation in GBM patients with IDH1/2 mutations. These mutations led to a hypermethylation phenotype and alteration in cellular metabolism in response to hypoxic and oxidative stress ${ }^{17}$. However, as of now, the ability to target GBMs with IDH mutations remains limited, and more investigations are necessary to optimize the therapeutic strategies targeting IDH mutant subsets of GBMs.

\section{Glutamine metabolism}

In addition to glucose, amino acids can also act as a fuel for cancer cells and funnel into the tricarboxylic acid 
cycle $(\text { TCA })^{18}$. Glutamine is hydrolyzed to glutamate by glutaminase (GLS) and is further converted to ?ketoglutarate (?-KG) that finally enters the TCA cycle. In the presence of mutant IDH1, ?- ketoglutarate is converted to D-2-hydroxyglutarate (2-HG) thus affecting the TCA cycle ${ }^{19}$. The NMR spectra showed that the glutamine concentration was significantly higher in GBM patients than in control subjects ${ }^{20}$. The conversion of glutamate to glutamine by glutamine synthetase (GS) is compromised in GBM patients that improved their survival rate ${ }^{21}$. In GBM cell cultures, it has been reported that around $60 \%$ of the glutamine is converted to alanine ${ }^{22}$. Glutaminolysis also generates $\mathrm{NADPH}$ which is used for fatty acid biosynthesis via the activity of malate dehydrogenase.

\section{Glutaminase}

Phosphate-activated glutaminase, the enzyme converting glutamine to glutamate plays a remarkable role in tumor biology. There are two genes encoding for GLS: the GLS encoding kidney-type (K-type) isozymes and GLS2 encoding liver-type (L-type) isozymes. The L-type is expressed in brain. Brain tumors show differential expression of GLS transcripts depending on their cellular origin. GLS isoforms play opposite roles in tumorigenesis, as the expression of K-type is correlated with greater cell proliferation while that of L-type with low proliferation and quiescent/resting cells. Knocking down GLS in GBM cells led to the reversion of the transformed phenotype. The similar effects were obtained by the overexpression of GLS2 gene in $\mathrm{GBM}^{23}$.

\section{Lipid metabolism}

Lipids, such as fatty acids, cholesterol, triglycerides, cholesterol esters, phospholipids and spingolipids are the important component of biological membranes. Other than their role as structural component, they also function as energy resource and as signaling molecules to maintain cell growth. Lipid metabolism is largely impaired in cancers. In GBM, higher levels of unsaturated fatty acids are observed compared with normal brain. Key proteins such as SREBP-1, Acetyl-CoA carboxylase (ACC), Fatty acid synthase (FAS) and low-density lipoprotein receptor (LDLR) are up-regulated in GBMs. The oncogenic signaling pathway EGFR/PI3K/Akt regulates the lipid metabolic reprogramming. Recent studies have shown that fatty acyl-CoA synthetase VL3 (ACSVL3) is involved in maintenance of GBM stem cell and in their tumorinitiating capacity in neurospheres ${ }^{24}$.
Sterol Regulatory Element-Binding Protein 1 (SREBP-1)

SREBP-1 is the transcription factor that regulates de novo fatty acid synthesis. It has three isoforms SREBP$1 \mathrm{a},-1 \mathrm{c}$ and -2 that play differential role in regulation of lipid metabolism. SREBP-1 plays a major role in energy metabolism including fatty acid and glucose metabolism while, SREBP-2 activates cholesterol synthesis. SREBPs are synthesized in inactive form bound to ER. These are activated by sequential proteolytic cleavage and translocate to Golgi complex. Eventually, their N-terminal domain is released into the nucleus where it activates the target genes. It was observed that SREBP-1 is highly up-regulated in GBM cell lines and its $\mathrm{N}$-terminal domain is present in the nucleus of tumor cells in patients' tissues ${ }^{25}$. Inhibition or reduction of SREBP-1 by pharmacologic agents significantly induced GBM cell death ${ }^{26}$. Thus, SREBP-1 is considered a promising molecular target for GBM therapy.

\section{Low-density lipoprotein receptor (LDLR):}

Cholesterol is the major component in cell membranes. Extra cholesterol is esterified to form cholesterol esters. Maintenance of cholesterol levels is important for cell morphology and its function. In human blood, cholesterol is transported from liver to the rest of the body by lipoproteins such as low density lipoproteins (LDL). The LDLR is a cell surface protein that binds LDL and transports it inside the cell. LDLR is found to be highly expressed in GBM ensures that tumor cells obtain sufficient cholesterol for their rapid growth and division. Its upregulation could explain the accumulation of cholesterol esters in GBM. LDLR is shown to be upregulated by EGFR/PI3K/Akt pathway which was found to be mediated by SREBP-1 and not -2 in GBM cells ${ }^{26}$.

\section{Pentose Phosphate Pathway (PPP)}

Glucose is a common fuel entering cells through a glucose transporter and being phosphorylated to form glucose6-phosphate (G-6-P), which can be further metabolized by both glycolysis and the PPP. PPP has oxidative and the non-oxidative pathway with G6PD, transketolase and transaldolase catalyzing their rate limiting steps. Glycolysis and Gluconeogenesis coordinate with the PPP to control the production of NADPH and Ribose 5-Phosphate (R-5-P), which determines whether the oxidative or non-oxidative pathway of PPP would be activated. GBM shows a pentose phosphate flux rate of approximately $4 \%$ of the total glucose flux. Both the activity and the regulation of glucose-6-phosphate dehydrogenase (G6PDH) are altered in $\mathrm{GBMs}^{27}$. It was recently reported that the PPP enzyme expression increased in rapidly dividing GBM cells, whereas 
glycolytic enzymes were elevated in migrating cells ${ }^{28}$. It was also reported that the knockdown of G6PD reduced GBM cell proliferation while that of ALDOC (encoding Aldolase c) knockdown decreased migration ${ }^{28}$.

\section{Conclusion}

Metabolic reprogramming is a key feature of oncogenesis and the recent studies have revealed that the glucose, glutamine and lipid metabolism are largely impaired in GBM facilitating malignancy while the role of PPP still remains obscured. Targeting molecules and enzymes that metabolically reprogram GBM can be a novel and potential therapeutic approach. Further understanding of the metabolic alterations in GBM will helps in developing more promising approaches to abrogate GBM malignancy and to overcome GBM resistance to current therapeutic approaches.

\section{REFERENCES}

1. Ramirez YP, Weatherbee JL, Wheelhouse RT, Ross AH. Glioblastoma multiforme therapy and mechanisms of resistance. Pharmaceuticals (Basel). 2013; 6:1475-506.

2. Ru P, Williams TM, Chakravarti A, Guo D. Tumor metabolism of malignant gliomas. Cancers (Basel). 2013; 5:1469-84.

3. Warburg 0, Wind F, Negelein E. The metabolism of tumors in the body. J Gen Physiol.1927; 8:519-30.

4. Parliament MB, Franko AJ, Allalunis-Turner MJ, Mielke BW, Santos CL, et al. Anomalous patterns of nitroimidazole binding adjacent to necrosis in human glioma xenografts: possible role of decreased oxygen consumption. Br J Cancer. 1997; 75:311-8.

5. Franko AJ, Parliament MB, Allalunis-Turner MJ, Wolokoff BG. Variable presence of hypoxia in M006 human glioma spheroids and in spheroids and xenografts of clonally derived sublines. Br J Cancer. 1998; 78:1261-8.

6. Allalunis-Turner MJ, Franko AJ, Parliament MB. Modulation of oxygen consumption rate and vascular endothelial growth factor mRNA expression in human malignant glioma cells by hypoxia. Br J Cancer. 1999; 80:104-9.

7. DeBerardinis RJ, Mancuso A, Daikhin E, Nissim I, Yudkoff M, et al. Beyond aerobic glycolysis: transformed cells can engage in glutamine metabolism that exceeds the requirement for protein and nucleotide synthesis. Proc Natl Acad Sci U S A. 2007; 104:19345-50.

8. Wise DR, DeBerardinis RJ, Mancuso A, Sayed N, Zhang XY, et al. Myc regulates a transcriptional program that stimulates mitochondrial glutaminolysis and leads to glutamine addiction. Proc Natl Acad Sci U S A. 2008; 105:18782-7.

9. Wolf A, Agnihotri S, Micallef J, Mukherjee J, Sabha N, et al. Hexokinase 2 is a key mediator of aerobic glycolysis and promotes tumor growth in human glioblastoma multiforme. J Exp Med. 2011; 208:313-26.

10. Agnihotri S, Wolf A, Munoz DM, Smith CJ, Gajadhar A, et al. A GATA4-regulated tumor suppressor network represses formation of malignant human astrocytomas. J Exp Med. 2011; 208:689-702.

11. Mazurek S, Boschek CB, Hugo F, Eigenbrodt E. Pyruvate kinase type M2 and its role in tumor growth and spreading. Semin Cancer Biol. 2005;15:300-8.

12. Yang W, Zheng Y, Xia Y, Ji H, Chen X, et al. ERK1/2-dependent phosphorylation and nuclear translocation of PKM2 promotes the Warburg effect. Nat Cell Biol. 2012; 14:1295-304.

13. Yang W, Xia Y, Ji H, Zheng Y, Liang J, et al. Nuclear PKM2 regulates $\beta$-catenin transactivation upon EGFR activation. Nature. 2011; 480:118-22.

14. Yang W, Xia Y, Cao Y, Zheng Y, Bu W, et al. EGFR-induced and PKC\& monoubiquitylation-dependent NF-KB activation upregulates PKM2 expression and promotes tumorigenesis. Mol Cell. 2012; 48:771-84.

15. Mukherjee J, Phillips JJ, Zheng S, Wiencke J, Ronen SM, et al. Pyruvate kinase M2 expression, but not pyruvate kinase activity, is up-regulated in a grade-specific manner in human glioma. PLOS One. 2013;8:e57610.

16. Morfouace M, Lalier L, Oliver L, Cheray M, Pecqueur C et al. Control of glioma cell death and differentiation by PKM2-0ct4 interaction. Cell Death Dis. $2014 ; 5: e 1036$.

17. Koh J, Cho H, Kim H, Kim SI, Yun S, et al. IDH2 mutation in gliomas including novel mutation. Neuropathology. 2015; 35:236-44.

18. Tanaka K, Sasayama T, Irino Y, Takata K, Nagashima H, et al. Compensatory glutamine metabolism promotes glioblastoma resistance to mTOR inhibitor treatment. J Clin Invest. $2015 ; 125: 1591-602$.

19. Ohka F, Ito M, Ranjit M, Senga T, Motomura A, et al. Quantitative metabolome analysis profiles activation of glutaminolysis in glioma with IDH1 mutation. Tumour Biol. 2014; 35:5911-20.

20. Kallenberg K, Bock HC, Helms G, Jung K, Wrede A, et al. Untreated glioblastoma multiforme: increased myo-inositol and glutamine levels in the contralateral cerebral hemisphere at proton MR spectroscopy. Radiology. 2009; 253:805-12.

21. Rosati A, Marconi S, Pollo B, Tomassini A, Lovato L, et al. Epilepsy in glioblastoma multiforme: correlation with glutamine synthetase levels. J Neurooncol. 2009; 93:319-24.

22. DeBerardinis RJ, Mancuso A, Daikhin E, Nissim I, Yudkoff M, et al. Beyond aerobic glycolysis: transformed cells can engage in glutamine metabolism that exceeds the requirement for protein and nucleotide synthesis. Proc Natl Acad Sci U S A. 2007; 104:19345-50.

23. Cheng T, Sudderth J, Yang C, Mullen AR, Jin ES, et al. Pyruvate carboxylase is required for glutamine-independent growth of tumor cells. Proc Natl Acad Sci U S A. 2011; 108:8674-9.

24. Sun P, Xia S, Lal B, Shi X, Yang KS, et al. Lipid metabolism enzyme ACSVL3 supports glioblastoma stem cell maintenance and tumorigenicity. BMC Cancer. 2014 ; 14:401.

25. Guo D, Prins RM, Dang J, Kuga D, Iwanami A, et al. EGFR signaling through an Akt-SREBP-1-dependent, rapamycin-resistant pathway sensitizes glioblastomas to antilipogenic therapy. Sci Signal. 2009; 2:ra82.

26. Guo D, Reinitz F, Youssef M, Hong C, Nathanson D, et al. An LXR agonist promotes glioblastoma cell death through inhibition of an EGFR/AKT/SREBP-1/LDLR-dependent pathway. Cancer Discov. 2011; 1:442-56.

27. Loreck DJ, Galarraga J, Van der Feen J, Phang JM, Smith BH, et al. Regulation of the pentose phosphate pathway in human astrocytes and gliomas. Metab Brain Dis. 1987;2:31-46.

28. Kathagen-Buhmann A, Schulte A, Weller J, Holz M, Herold-Mende C, et al. Glycolysis and the pentose phosphate pathway are differentially associated with the dichotomous regulation of glioblastoma cell migration versus proliferation. Neuro Oncol. 2016 Feb 24. pii: now024. [Epub ahead of print] 